\title{
Impact of Sustained Weight Loss Achieved through Roux-en-Y Gastric Bypass or a Lifestyle Intervention on Ghrelin, Obestatin, and Ghrelin/Obestatin Ratio in Morbidly Obese Patients
}

\author{
Catia Martins • Louise Kjelstrup • Ingrid L. Mostad • \\ Bård Kulseng
}

Published online: 13 April 2011

(C) The Author(s) 2011. This article is published with open access at Springerlink.com

\begin{abstract}
Background Appetite-regulating hormones seem to play an important role in weight loss after bariatric surgery. Less is known regarding long-term weight loss maintenance. The objective of the study was to evaluate ghrelin and obestatin levels following long-term weight loss achieved through bariatric surgery or a lifestyle intervention in morbidly obese patients.

Methods The study was cross-sectional in design carried out in a university research center setting. The participants were weight-stable morbidly obese patients who had undergone, on average, 3 years ago, Roux-en-Y gastric bypass (RYGB) surgery $(n=9)$ or a lifestyle weight loss intervention $(n=8)$, and patients on a waiting list for bariatric surgery (control group; $n=9$ ). The main outcome
\end{abstract}

C. Martins $\cdot$ I. L. Mostad

Faculty of Medicine, Department of Cancer

Research and Molecular Medicine, Norwegian University of

Science and Technology,

Trondheim, Norway

C. Martins $(\square) \cdot$ B. Kulseng

Centre for Obesity, Department of Surgery,

St. Olavs Hospital-Trondheim University Hospital,

Trondheim, Norway

e-mail: catia.martins@ntnu.no

L. Kjelstrup · I. L. Mostad

Division of Clinical Nutrition, Department of Clinical Service,

St. Olavs Hospital,

Trondheim, Norway

L. Kjelstrup

Department of Human Nutrition, University of Copenhagen,

Copenhagen, Denmark measures were fasting/postprandial plasma levels of total ghrelin and obestatin and ghrelin/obestatin ratio.

Results Fasting ghrelin and obestatin plasma levels were significantly elevated in the RYGB, but not in the lifestyle group, as compared with the control group. There was no statistical significant difference in fasting ghrelin/obestatin ratio among study groups. Ghrelin levels were suppressed after breakfast in all groups, with no significant differences in postprandial levels overtime between them. Obestatin levels did not change postprandially in any of the groups, but the area under the curve was significantly higher in the RYGB than in the control group.

Conclusions Sustained weight loss maintenance seems to be associated with increased fasting levels of ghrelin and obestatin after RYGB surgery, but not after a lifestyle intervention, while maintaining ghrelin/obestatin ratio. Ghrelin is, therefore, unlikely to contribute to weight loss maintenance after RYGB, and other mechanisms are probably involved.

Keywords Weight loss maintenance - Bariatric surgery Lifestyle intervention $\cdot$ Ghrelin $\cdot$ Obestatin

\section{Introduction}

The impact of weight loss achieved through Roux-en-Y gastric bypass (RYGB) on the plasma levels of ghrelin, the only peripheral hormone with orexigenic properties (i.e., having a stimulating effect on appetite) [1], remains a matter of continued debate. Cummings and colleagues [2] in 2002 were the first to report reduced ghrelin levels in patients who had undergone RYGB, compared with normal-weight and obese matched controls. This was a 
surprising finding because it suggested that RYGB reduced ghrelin secretion, while conventional methods of weight loss involving diet and exercise induced the opposite response [2-4]. This could contribute to the long-term weight loss maintenance observed with this bariatric procedure as compared with lifestyle interventions. However, subsequent studies have produced controversial results, with some reporting a significant reduction [5-8]; others, no change $[9,10]$; and others, an increase in fasting ghrelin plasma levels after RYGB [11, 12]. The status of energy balance (whether patients are in active weight loss or have achieved a stable lower body weight) could be a factor modulating ghrelin plasma levels after RYGB. Ghrelin plasma levels were reported to increase after RYGB in those undergoing active weight loss, but reported not to change in those who were weight-stable, despite a similar degree of weight loss [13]. However, other studies have shown that, despite patients actively losing weight 6 months after RYGB, their ghrelin plasma levels were significantly reduced compared with preoperative levels $[5,6]$. Overall, it remains unknown if long-term weight loss maintenance in morbidly obese patients after RYGB or a lifestyle intervention has any impact on ghrelin secretion.

Obestatin is a recently discovered 23-amino acid peptide encoded by the ghrelin gene [14]. Although in the original work of Zhang et al. [14] obestatin has been shown to suppress food intake and decrease gastric emptying, therefore antagonizing the orexigenic effect of ghrelin, subsequent studies in rodents have yielded controversial results [15-17]. In view of this controversy, it is important to understand how changes in energy balance affect obestatin plasma levels in humans. The majority [1820], but not all studies [21], show that fasting obestatin plasma levels are reduced in obese compared with normalweight individuals, therefore, supporting a role for obestatin in the regulation of body weight and energy homeostasis. Ghrelin/obestatin ratio has also been described to be reduced in obese women compared with normal-weight controls [21]. In contrast to the large number of studies on the impact of RYGB on ghrelin plasma levels, only one study has assessed the potential effects of this bariatric procedure on obestatin levels, reporting no change postoperatively at 2 years and a significant reduction in ghrelin/obestatin ratio [8]. To our knowledge, only two studies have looked at the impact of weight loss achieved through conservative means on obestatin plasma levels and they were both in children $[22,23]$. The aim of this study was, therefore, to determine the impact of sustained weight loss 3 years after RYGB or a lifestyle intervention on the fasting and postprandial plasma levels of ghrelin and obestatin and ghrelin/obestatin ratio in morbidly obese patients.

\section{Subjects and Methods}

\section{Participants}

Three groups of adult patients were recruited to participate in this study: (1) morbidly obese patients who had undergone, on average, 3 years before, RYGB surgery ( $n=$ 9); (2) or a lifestyle weight loss intervention $(n=8)$; and (3) morbid obese patients on waiting list for bariatric surgery $(n=9)$. We used the records from our center to identify the potential candidates, who were later contacted and invited to participate. The interval between intervention and metabolic evaluation was $36 \pm 4$ months for the RYGB group and $32 \pm$ 10 months for the lifestyle group. The exclusion criteria included pregnancy, enrolment in another obesity treatment, previous bariatric surgery, drug or alcohol abuse, and mental disorders and/or physical impairment which could interfere with the ability to comply with treatment. All the subjects were white Caucasian and with ages ranging from 25 to 61 years. Patients in all three groups had stable weight at the time of the evaluation, as defined by a change of no more than $5 \%$ in body weight during the preceding 3 months. Preintervention excess weight (in comparison with ideal weight) and body mass index (BMI) were calculated for each group. Weight loss at 3 years was expressed in absolute terms and as a percent of excess weight loss (EWL). EWL was calculated as (weight at baseline - weight at 3 years)/ (weight at baseline - ideal weight). The ideal weight was that associated with a BMI of $25 \mathrm{~kg} / \mathrm{m}^{2}$.

This study was conducted according to the guidelines laid down in the Declaration of Helsinki. All participants gave written consent before enrolling in the study, and approval was obtained from the regional Ethics Committee (Central Norway, Trondheim, Norway).

The surgical procedure consisted of creating a 20- to $30-\mathrm{ml}$ gastric pouch along the lesser curvature of the stomach. Division of the vagal nerve and its branches was avoided. The jejunum was then advanced in an antecolic position to create a $100-150-\mathrm{cm}$ long alimentary limb, which was anastomosed to the gastric pouch. All the procedures were performed laparoscopically and by the same team of surgeons.

The lifestyle intervention consisted of a "continuous care" multidisciplinary program at Røros Rehabilitation Centre (Norway), with intermittent stays at the center. The program focused on physical activity, healthy eating, and dynamic group-based psychotherapy [24].

\section{Protocol}

Participants arrived at the Research Center at St. Olav Hospital in the morning after an overnight fast (at least $12 \mathrm{~h}$ ) and were weighed without shoes and with light 
clothing. An intravenous cannula was inserted into an antecubital vein by a specialized nurse. Participants ingested a chilled milkshake test meal (Fresubin ${ }^{\circledR}$ 2-kcal drink $(200 \mathrm{ml}), 400 \mathrm{kcal}, 15.6 \mathrm{~g}$ fat $(35 \%), 45 \mathrm{~g}$ carbohydrates $(45 \%)$, and $20 \mathrm{~g}$ of protein $(20 \%)$ from Fresenius Kabi) within 10 min. Venous blood was drawn from the cannula before the meal and at 30,60, 90, and $120 \mathrm{~min}$ after meal consumption.

\section{Hormone Measurement}

Venous blood was collected into potassium/EDTA-coated tubes containing $500 \mathrm{KIU}$ aprotinin (Pentapharm, Basel, Switzerland)/ml whole blood). Samples were then centrifuged at $2,000 \times \mathrm{g}$ for $10 \mathrm{~min}$ and plasma kept at $-80^{\circ} \mathrm{C}$ for later analyses. Ghrelin and obestatin plasma levels were determined by radioimmunoassay (ghrelin Total RIA Kit ${ }^{\circledR}$, Linco Research, St. Charles, MO, USA and obestatin ultrasensitive RIA Kit ${ }^{\circledR}$, Phoenix Pharmaceuticals, 330 Beach Road, Burlingame, CA, USA, respectively). The sensitivity of the assays was of $93 \mathrm{pg} / \mathrm{ml}$ for ghrelin and $50 \mathrm{pg} / \mathrm{ml}$ for obestatin, with an inter-assay $\mathrm{CV}<10 \%$. Due to expected low concentrations of obestatin in these samples, they were previously concentrated by freezedrying. The obestatin and ghrelin reagents are highly specific without cross-reacting with other gastrointestinal hormones. All samples were assayed in duplicate.

\section{Statistical Analysis}

Statistical analysis was carried out using SPSS 15.0 (SPSS Inc., Chicago, IL). Data is reported as mean \pm SEM, and statistical significance was assumed at $P<0.05$, unless otherwise stated. Differences in the fasting plasma levels of ghrelin and obestatin, and ghrelin/obestatin ration, were assessed by one-way analysis of variance (ANOVA). The effect of group and time on the plasma levels of ghrelin and obestatin were assessed by a mixed between/within subjects ANOVA and changes overtime within groups were assessed by a repeated-measure ANOVA. The areas under the curve (AUC) for ghrelin and obestatin plasma levels were calculated from before to $120 \mathrm{~min}$ after breakfast, using the trapezoidal rule. The effect of group on the AUC for ghrelin and obestatin was assessed using one-way ANOVA. The Bonferroni adjustment was applied in all post hoc analysis $(P<0.01$ was used to account for the possibility of an increased type 1 error). The magnitude of suppression of ghrelin and obestatin was calculated, for each participant, as peak (fasting plasma level) minus nadir (lower value in the postprandial state) and expressed as a percentage of baseline levels. Pearson correlations were used to test the relationship between ghrelin plasma levels and weight loss in groups (1) and (2).

\section{Results}

\section{Characteristics of Study Groups}

Table 1 shows the characteristics of each study group. There were significant differences in age among groups $(P<0.05)$, however, post hoc comparisons revealed no significant differences between specific groups, and no significant correlations were observed between age and fasting or AUC ghrelin or obestatin or ghrelin/obestatin ratio. There were no significant differences in preintervention excess weight or BMI between the three groups. The RYGB group lost on average $34.0 \pm 20.8 \mathrm{~kg}$ $(P<0.001)$, while the lifestyle group lost $7.2 \pm 5.2 \mathrm{~kg}(P<$ $0.001)$, a difference that was statistically significant $(P<$ 0.01). EWL (in percent) was also much larger in the RYGB compared with the lifestyle group $(P<0.0001)$. Postintervention BMI in the RYGB group was significantly lower compared with the control group $(P<0.05)$, but there were no statistically significant differences between the RYGB and lifestyle groups or between the lifestyle and control groups.

Fasting Plasma Levels of Ghrelin and Obestatin and Ghrelin/Obestatin Ratio

Fasting plasma levels of ghrelin and obestatin and ghrelin/ obestatin ratio in the different groups can be seen in Table 2. There was a significant difference in fasting ghrelin plasma levels among groups $(P<0.01)$. Post hoc comparisons indicated that fasting ghrelin plasma levels were significantly elevated in the RYGB compared with the pre-operative group $(P=0.002)$, while there were no significant differences between the lifestyle and control groups or between the RYGB and the lifestyle groups.

There was also a significant difference in fasting obestatin plasma levels among groups $(P<0.01)$. Post hoc comparisons revealed that, compared with the control group, the RYGB group exhibited significantly higher fasting obestatin plasma levels $(P=0.002)$, while there were no significant differences between the lifestyle and control groups or between the RYGB and the lifestyle groups.

There was no statistically significant difference in fasting ghrelin/obestatin ratio among the different study groups $(P>0.05)$.

\section{Postprandial Plasma Levels of Ghrelin and Obestatin}

Analysis of variance revealed a significant main effect of time $(P<0.0001)$ and group $(P=0.045)$, but no time $\times$ group interaction on ghrelin plasma levels (Fig. 1a). However, post hoc comparisons indicated no significant differences in postprandial ghrelin plasma levels overtime between the 
Table 1 Participant's characteristics

\begin{tabular}{lccc}
\hline & RYGB $(n=9)$ & Lifestyle $(n=8)$ & Control $(n=9)$ \\
\hline Age (years) & $42 \pm 10$ & $51 \pm 7$ & $38 \pm 8$ \\
Pre-intervention excess weight $(\mathrm{kg})$ & $62.4 \pm 19.8$ & $54.1 \pm 14.7$ & $58.4 \pm 12.4$ \\
Pre-intervention BMI $\left(\mathrm{kg} / \mathrm{m}^{2}\right)$ & $46.6 \pm 6.6$ & $44.6 \pm 6.2$ & $43.9 \pm 3.1$ \\
Post-intervention BMI $\left(\mathrm{kg} / \mathrm{m}^{2}\right)$ & $35.0 \pm 4.5^{*}$ & $42.1 \pm 7.0$ & $43.9 \pm 3.1^{*}$ \\
Weight loss at 3 years $(\mathrm{kg})$ & $34.0 \pm 20.8^{*}$ & $7.2 \pm 5.2^{*}$ & - \\
Excess weight loss $(\%)$ & $51.9 \pm 17.4^{* *}$ & $14.4 \pm 12.2^{* *}$ & - \\
\hline
\end{tabular}

Results are expressed as mean $\pm \mathrm{SD}$

$* P<0.01$, with means sharing the same symbol denoting significant differences between groups

$* * P<0.0001$, with means sharing the same symbol denoting significant differences between groups

three groups. When each group was analyzed separately, a significant effect of time on ghrelin plasma levels was observed in the RYGB $(P<0.01)$ and control group $(P=$ $0.022)$ and a tendency in the lifestyle group $(P=0.058)$. The area under the curve for ghrelin $\left(0-120 \mathrm{~min} ; \mathrm{pg} / \mathrm{ml} \mathrm{min}^{-1}\right)$ in the three study groups can be seen in Fig. 1b. ANOVA showed no overall main effect of group $(P>0.05)$. The magnitude of suppression of total ghrelin in response to the test meal was comparable in all groups at $28 \pm 18 \%, 22 \pm$ $13 \%$, and $23 \pm 9 \%$, in the RYGB, lifestyle and control groups, respectively $(P>0.05)$.

A significant main effect of time $(P<0.05)$ and group $(P<0.05)$ and a time $\times$ group interaction $(P<0.05)$ was observed on obestatin plasma levels (Fig. 2a). Post hoc comparisons revealed no significant differences in obestatin plasma levels overtime between groups, despite a tendency for higher postprandial levels in the RYGB group compared with the control group $(P=0.021)$. When each group was analyzed separately, no significant effect of time was observed on obestatin plasma levels in any group. The area under the curve for obestatin $\left(0-120 \mathrm{~min} ; \mathrm{pg} / \mathrm{ml} \mathrm{min}^{-1}\right)$ in the three study groups can be seen in Fig. 2b. ANOVA showed an overall main effect of group $(P=0.012)$, and post hoc analysis revealed that obestatin AUC was higher in the RYGB group compared with the pre-operative control group $(P=0.01)$. The magnitude of suppression of obestatin in response to the test meal was comparable in all groups at $33 \pm 13 \%, 25 \pm 22 \%$, and $17 \pm 13 \%$, in the RYGB, lifestyle, and control groups $(P>0.05)$.
Correlations between Weight Loss, Ghrelin and Obestatin Plasma Levels, and Ghrelin/Obestatin Ratio

Correlations between weight loss at 3 years in the RYGB and lifestyle groups and ghrelin, obestatin, and TG/ obestatin ratio were performed. Two outliers were identified in the RYGB group (weight loss of 76 and $61.2 \mathrm{~kg}$ ( 3 and 2.2 SDs away from the mean, respectively)). No significant correlations were observed between weight loss and ghrelin or obestatin levels (fasting or AUC) or ghrelin/obestatin ratio, either when all the patients' data was used or after removing the two outliers.

\section{Discussion}

In the present study, we found that fasting ghrelin and obestatin plasma levels in weight-stable patients 3 years after RYGB, but not after a lifestyle intervention, were significantly higher compared with pre-operative morbid obese patients (control group), despite no difference in ghrelin/obestatin ratio among groups.

Ghrelin levels have been shown to be down-regulated in obese individuals $[25,26]$ and to increase in response to diet- and/or exercise-induced weight loss [2-4], suggesting that ghrelin plays a role in the long-term regulation of energy balance. An increase in ghrelin levels secondary to weight loss can be seen as a compensatory mechanism to try to restore energy balance by upregulating hunger levels

Table 2 Fasting plasma levels of ghrelin and obestatin and ghrelin/obestatin ratio in the different study groups

\begin{tabular}{lccr}
\hline & RYGB $(n=9)$ & Lifestyle $(n=8)$ & Control $(n=9)$ \\
\hline Ghrelin $(\mathrm{pmol} / \mathrm{L})$ & $729 \pm 218^{*}$ & $632 \pm 99$ & $449 \pm 114^{*}$ \\
Obestatin $(\mathrm{pg} / \mathrm{ml})$ & $102 \pm 37^{*}$ & $74 \pm 27$ & $48 \pm 18^{*}$ \\
Ghrelin/obestatin & $7.4 \pm 1.2$ & $9.2 \pm 2.9$ & $10.4 \pm 4.7$ \\
\hline
\end{tabular}

Results are expressed as mean $\pm \mathrm{SD}$

${ }^{*} P<0.01$, with means sharing the same symbol denoting significant differences between groups 

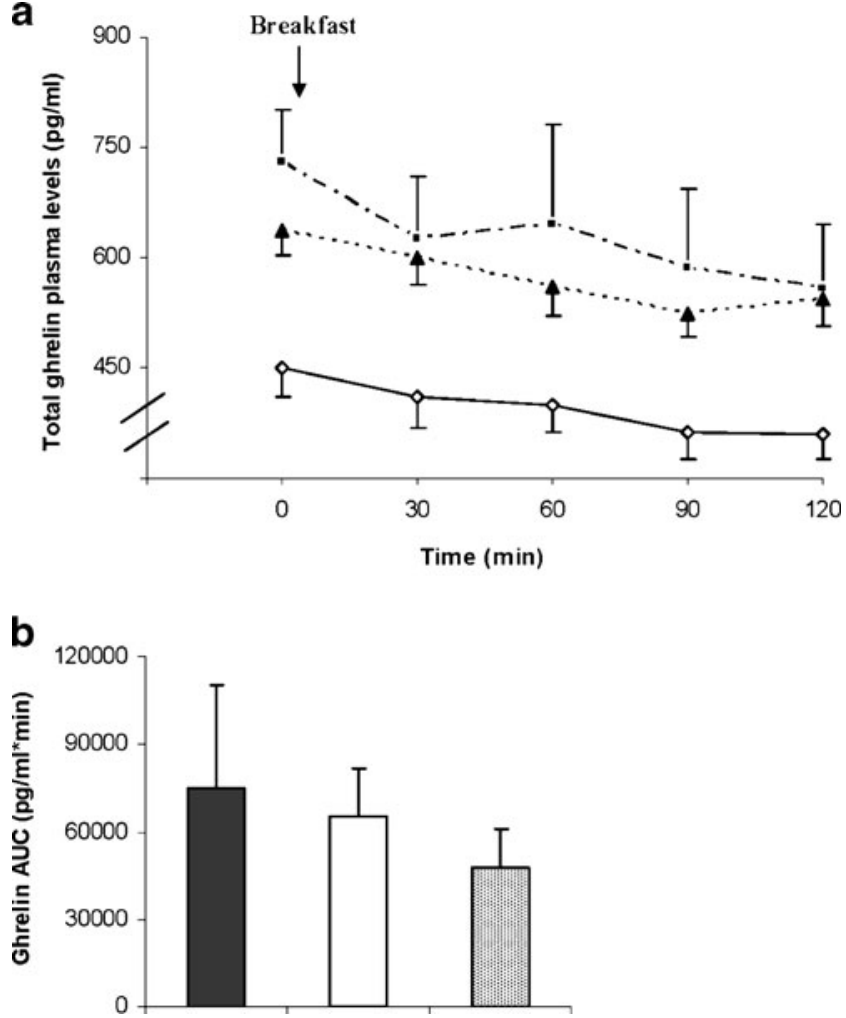

Fig. 1 a Ghrelin plasma levels (picograms per milliliter) over time after breakfast in pre-operative morbid obese patients $(n=9$; open diamonds) and patients 3 years after RYGB ( $n=9$; closed squares) or a lifestyle intervention ( $n=8 ;$ closed triangles). Values represent means \pm SEM. Repeated-measures ANOVA showed a significant effect of time $(P<$ $0.0001)$ and group $(P=0.045)$, but no interaction. b Area under the curve for ghrelin $(0-120 \mathrm{~min})$ (picograms per milliliter per minute) in pre-operative morbidly obese patients (hatched bar) and patients 3 years after RYGB (solid bar) or a lifestyle intervention (open bar). ANOVA showed no overall main effect of group $(P>0.05)$

and ultimately energy intake. Unexpectedly, weight loss after RYGB has been previously reported not to lead to a compensatory increase in ghrelin concentrations $[9,10]$ or even to induce a reduction in its levels [5-8]. This was, however, not supported by our results, which showed a significant increase in fasting ghrelin plasma levels 3 years after RYGB. It is important to acknowledge that the majority of the previously published studies have focused on short-term changes (from 6 weeks to 12 months), and it is likely that ghrelin response after RYGB changes overtime.

It is well known that changes on the plasma levels of ghrelin following bariatric surgery depend on the surgical procedure. Gastric bypass surgery markedly suppresses ghrelin concentrations while restrictive techniques such as adjustable gastric banding lead to an increase [5]. These differences in ghrelin response have been shown to be independent of weight loss and a direct result of the degree of functionality of the fundus [5,27], the anatomical region which has been shown to be the richest source of ghrelin production in humans [28]. It is rather intriguing, therefore, that despite bypassing most of the stomach and preventing the fundus from contact with nutrients, circulating ghrelin plasma levels were increased 3 years after RYGB in the present study. It is possible that ghrelin-secreting cells in the stomach increase the production of ghrelin as a counterregulatory mechanism to the long-term absence of contact with nutrients as a result of RYGB. Another possibility is that the increase in ghrelin levels reflects an increased secretion from ghrelin-secreting cells outside of the stomach, since it is known that other organs, including the duodenum, the jejunum, and the lung can secret ghrelin [29]. However, the exact cause for the increased ghrelin levels after long-term sustained weight loss following RYGB remains a matter of continued discussion, and more studies are needed in this area. The only available study
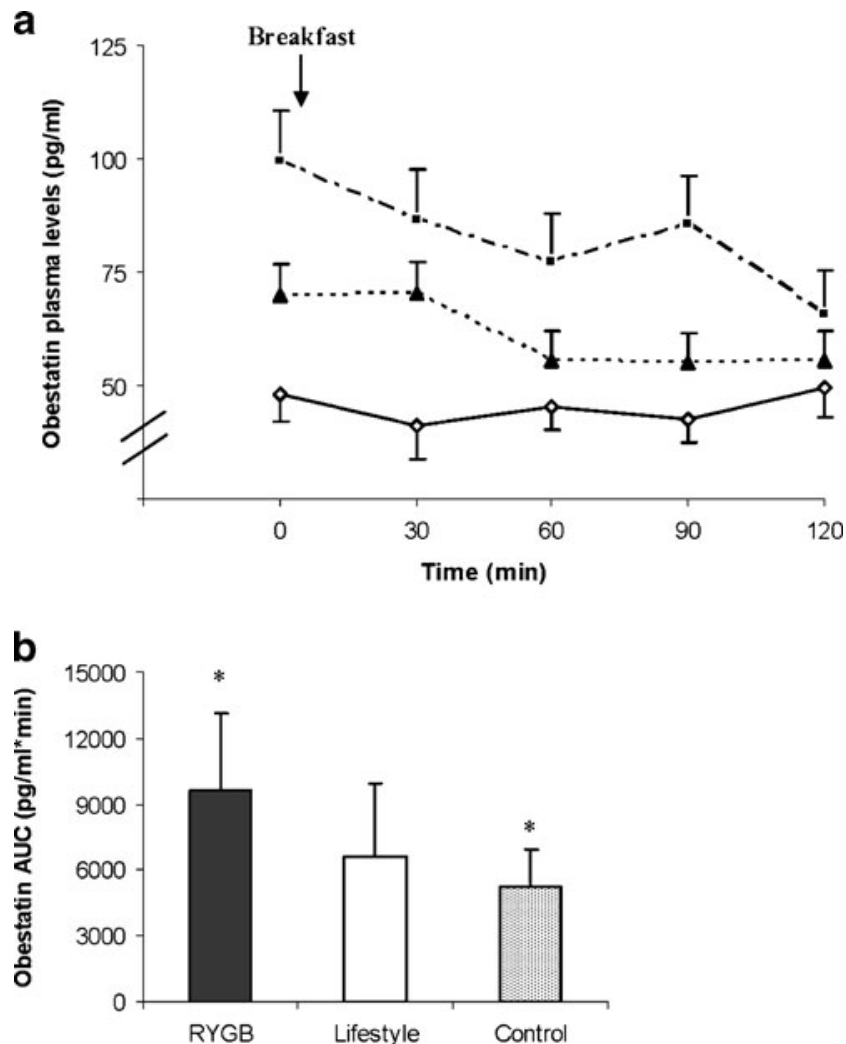

Fig. 2 a Obestatin plasma levels (picograms per milliliter) over time after breakfast in pre-operative morbid obese patients $(n=9$; open diamonds) and patients three years after RYGB ( $n=9$; closed squares) or a lifestyle intervention ( $n=8$; closed triangles). Values represent means \pm SEM. Repeated-measures ANOVA showed a significant effect of time $(P<0.05)$ and group $(P<0.05)$ and a time $\times$ group interaction $(P<0.01)$. b Area under the curve for obestatin $(0-120 \mathrm{~min}$; picograms per milliliter per minute) in pre-operative morbidly obese patients (hatched bar) and patients 3 years after RYGB (solid bar) or a lifestyle intervention (open bar). ANOVA showed an overall main effect of group on obestatin AUC $(P=0.007)$. Asterisk reveals significant differences between groups $(P=0.01)$ 
with a similar follow-up to ours (approximately 3 years) reported no difference in the fasting plasma levels of either total ghrelin or its active component (acylated ghrelin) between weight-stable patients after RYGB and BMI- and age-matched controls [28]. However, that study suffers from several drawbacks since there was no pre-operative control group and the time after RYGB was very heterogeneous (15-71 months) [28]. Differences in surgical techniques (such as size of the pouch and integrity of the remaining fundus), follow-up periods, and magnitude of weight loss, as well as the status of energy balance are likely to contribute to some of the inconsistencies reported above. Although a reduction in ghrelin levels shortly after RYGB can contribute to weight reduction, the present study seems to suggest that ghrelin is unlikely to be a mediator in long-term weight loss maintenance after RYGB, particularly because we found no correlation between ghrelin levels at 3 years and weight loss.

Fasting ghrelin plasma levels in the lifestyle group, on the other hand, were not significantly different from the preoperative control group. Three potential explanations can be provided for this finding. First, it is possible that the weight reduction was not large enough to have produced a significant increase in ghrelin plasma levels. Second, it is possible that ghrelin levels had previously been increased but that they returned to baseline levels afterwards, since at least two studies have shown that ghrelin plasma levels return to baseline with sustained weight loss maintenance after lifestyle interventions [30, 31]. Finally, despite a significant weight loss, BMI at 3 years in the lifestyle group was not significantly different from the BMI of the control group.

Ghrelin also seems to reflect acute feeding state in humans, since its concentrations increase with fasting and quickly decline after a meal [29] and have been associated with pre-meal hunger and meal initiation [32]. The postprandial suppression of ghrelin seen postprandially in normal-weight individuals [2] has been found to be blunted after RYGB in some studies [2, 6, 9, 33] while exaggerated in others [34]. In the present study, ghrelin plasma levels decreased postprandially in all groups, with no significant differences in postprandial plasma levels or the magnitude of maximal suppression among them.

We have also reported in the present study that weight loss maintenance 3 years after RYGB, but not after a lifestyle intervention, was associated with a significant increase in fasting obestatin plasma levels, compared with pre-operative morbid obese patients. Moreover, no differences were observed in ghrelin/obestatin ratio among groups. These results are extremely exciting since the only previously published study in this area reported no change in fasting obestatin plasma levels 2 years after RYGB and a significant reduction in ghrelin/obestatin ratio [8]. Regarding lifestyle interventions, studies in children have reported increased obestatin plasma levels $[22,23]$ and ghrelin/obestatin ratio [22] after weight reduction achieved through diet and physical activity. However, studies in adults, and particularly in the morbidly obese population, are missing. The same potential reasons highlighted before for the absence of a significant difference in ghrelin levels between the lifestyle and the control group could also apply here for obestatin. More studies are needed to clarify the impact of weight loss and weight loss maintenance achieved through different methods on obestatin plasma levels.

The postprandial profile of obestatin remains also controversial. Most of the studies show that obestatin levels decrease in the postprandial period in both normal-weight $[18,20,35]$ and borderline obese individuals [20]; however, one study reported no change in obestatin plasma levels after a mixed meal in normal-weight and morbidly obese subjects [19]. We were the first to look at the impact of weight loss achieved by RYGB or lifestyle intervention on the postprandial release of obestatin. We have shown no significant change in obestatin plasma levels overtime after a liquid balanced meal in neither morbid obese patients (controls) nor weightstable patients 3 years after RYGB or a lifestyle intervention. However, postprandial obestatin levels were significantly higher in the RYGB group compared with the pre-operative control group. It is difficult to speculate on the implications of our findings, since, as highlighted by a recent review, the role of obestatin on energy balance remains controversial [36]. More studies are urgently needed to elucidate the role of obestatin on energy homeostasis.

This study has a few potential limitations. First, it has a cross-sectional design and a low sample size. We acknowledge that this study might not have adequate statistical power and, therefore, should be performed in larger cohorts before strong conclusions can be drawn regarding their clinical relevance. Moreover, more longitudinal studies need to be done to unveil the physiological mechanisms mediating longterm weight loss after RYGB and lifestyle interventions in this group of patients. Second, we measured total ghrelin and not acylated ghrelin, which is thought to be essential for its biological activities. Although total ghrelin has been shown to correlate well with acylated ghrelin [37], the two forms may be differentially regulated in altered nutritional states and pre- and post-gastric surgery, and therefore we cannot exclude the possibility that acylated ghrelin may have responded differently and shown a different relationship with obestatin. Third, there was an overall difference in age among groups. However, post hoc comparisons revealed no significant differences between specific groups, and no significant correlations were observed between age and fasting or AUC ghrelin or obestatin or ghrelin/obestatin ratio, making it unlikely for age differences to have had a significant impact on our results. Despite these limitations, we would like to emphasize that this study is strengthened by 
the existence of a pre-operative control group, the fact that patients were weight-stable and the long follow-up period.

In conclusion, the results of this study suggest that weight loss maintenance after RYGB is associated with a significant increase in fasting ghrelin and obestatin plasma levels, but no change in ghrelin/obestatin ratio, compared with pre-operative morbidly obese patients (controls). On the other hand, weight loss (maintenance) after a lifestyle intervention was not associated with a significant increase in fasting ghrelin or obestatin levels, although this was likely to have been caused by the much lower magnitude of weight loss (7 vs. $34 \mathrm{~kg}$ ). Ghrelin is, therefore, unlikely to contribute to long-term weight loss maintenance after RYGB and other factors are likely to be involved.

Acknowledgments We would like to thank Mrs. Sissel Salater and Mrs. Trude Synnøve Grande Langeng for their clinical assistance and all the research participants for their cooperation and commitment.

Conflict of interest The authors declare that there is no conflict of interest that would prejudice the impartiality of this scientific work.

Funding This project was supported by a grant from the Liaison Committee between Central Norway Regional Health Authority and the Norwegian University of Science and Technology in partnership with St. Olav Hospital (Trondheim, Norway).

Open Access This article is distributed under the terms of the Creative Commons Attribution Noncommercial License which permits any noncommercial use, distribution, and reproduction in any medium, provided the original author(s) and source are credited.

\section{References}

1. Wren AM, Seal LJ, Cohen MA, et al. Ghrelin enhances appetite and increases food intake in humans. J Clin Endocrinol Metab. 2001;86:5992-5.

2. Cummings DE, Weigle DS, Frayo RS, et al. Plasma ghrelin levels after diet-induced weight loss or gastric bypass surgery. N Engl J Med. 2002;346:1623-30.

3. Hansen TK, Dall R, Hosoda H, et al. Weight loss increases circulating levels of ghrelin in human obesity. Clin Endocrinol (Oxf). 2009;56:203-6.

4. Leidy HJ, Gardner JK, Frye BR, et al. Circulating ghrelin is sensitive to changes in body weight during a diet and exercise program in normal-weight young women. J Clin Endocrinol Metab. 2004;89:2659-64.

5. Fruhbeck G, Rotellar F, Hernandez-Lizoain J, et al. Fasting plasma ghrelin concentrations 6 months after gastric bypass are not determined by weight loss or changes in insulinemia. Obes Surg. 2004;14:1208-15.

6. Morinigo R, Casamitjana R, Moize V, et al. Short-term effects of gastric bypass surgery on circulating ghrelin levels. Obesity. 2004;12:1108-16.

7. Geloneze B, Tambascia MA, Pilla VF, Geloneze SR, Repetto EM, Pareja JC. Ghrelin: a gut-brain hormone: effect of gastric bypass surgery. Obes Surg. 2003;13:17-22.
8. Roth CL, Reinehr T, Schernthaner G-H, et al. Ghrelin and obestatin levels in severely obese women before and after weight loss after Roux-en-Y gastric bypass surgery. Obes Surg. 2009;19:29-35.

9. Foschi D, Corsi F, Colombo F, et al. Different effects of vertical banded gastroplasty and Roux-en-Y gastric bypass on meal inhibition of ghrelin secretion in morbidly obese patients. J Invest Surg. 2008;21:77-81.

10. Korner J, Inabnet W, Febres G, et al. Prospective study of gut hormone and metabolic changes after adjustable gastric banding and Roux-en-Y gastric bypass. Int J Obes. 2009;33:786-95.

11. Garcia-Fuentes E, Garrido-Sanchez L, Garcia-Almeida J, et al. Different effect of laparoscopic Roux-en-Y gastric bypass and open biliopancreatic diversion of scopinaro on serum PYY and ghrelin levels. Obes Surg. 2008;18:1424-9.

12. Holdstock C, Engstrom BE, Ohrvall M, et al. Ghrelin and adipose tissue regulatory peptides: effect of gastric bypass surgery in obese humans. J Clin Endocrinol Metab. 2003;88:3177-83.

13. Faraj M, Havel PJ, Phelis S, et al. Plasma acylation-stimulating protein, adiponectin, leptin, and ghrelin before and after weight loss induced by gastric bypass surgery in morbidly obese subjects. J Clin Endocrinol Metab. 2003;88:1594-602.

14. Zhang JV, Ren P-G, Avsian-Kretchmer O, et al. Obestatin, a peptide encoded by the ghrelin gene, opposes ghrelin's effects on food intake. Science. 2005;310:996-9.

15. Zizzari P, Longchamps R, Epelbaum J, et al. Obestatin partially affects ghrelin stimulation of food intake and growth hormone secretion in rodents. Endocrinology. 2007;148:1648-53.

16. Carlini VP, Schioth HB, de Barioglio SR. Obestatin improves memory performance and causes anxiolytic effects in rats. Biochem Biophys Res Commun. 2007;352:907-12.

17. Nogueiras R, Pfluger P, Tovar S, et al. Effects of obestatin on energy balance and growth hormone secretion in rodents. Endocrinology. 2007;148:21-6.

18. Lippl F, Erdmann J, Lichter N, et al. Relation of plasma obestatin levels to BMI, gender, age and insulin. Horm Metab Res. 2008;40:806-12.

19. Huda MSB, Durham BH, Wong SP, et al. Plasma obestatin levels are lower in obese and postgastrectomy subjects, but do not change in response to a meal. Int J Obes. 2008;32:129-35.

20. Guo ZF, Zheng X, Qin Y-W, et al. Circulating preprandial ghrelin to obestatin ratio is increased in human obesity. J Clin Endocrinol Metab. 2007;92:1875-80.

21. Vicennati V, Genghini S, De Iasio R, et al. Circulating obestatin levels and the ghrelin/obestatin ratio in obese women. Eur J Endocrinol. 2007;157:295-301.

22. Zou CC, Liang L, Wang CL, et al. The change in ghrelin and obestatin levels in obese children after weight reduction. Acta Paediatr. 2009;98:159-65.

23. Reinehr T, de Sousa G, Roth CL. Obestatin and ghrelin levels in obese children and adolescents before and after reduction of overweight. Clin Endocrinol (Oxf). 2008;68:304-10.

24. Martins C, Strømmen M, Stavne OA et al. Bariatric surgery versus lifestyle interventions for morbid obesity - changes in body weight, risk factors and comorbidities at 1 year. Obes Surg. 2010 (in press).

25. Tschop M, Weyer C, Tataranni PA, et al. Circulating ghrelin levels are decreased in human obesity. Diabetes. 2001;50:707-9.

26. Stylianou C, Galli-Tsinopoulou A, Farmakiotis D, et al. Ghrelin and leptin levels in obese adolescents. Relationship with body fat and insulin resistance. Hormones. 2007;6:295-303.

27. Fruhbeck G, Diez-Caballero A, Gil M, et al. The decrease in plasma ghrelin concentrations following bariatric surgery depends on the functional integrity of the fundus. Obes Surg. 2004;14:606-12.

28. Korner J, Bessler M, Cirilo LJ, et al. Effects of Roux-en-Y gastric bypass surgery on fasting and postprandial concentrations of plasma ghrelin, peptide YY, and insulin. J Clin Endocrinol Metab. 2005;90:359-65. 
29. Ariyasu H, Takaya K, Tagami T, et al. Stomach is a major source of circulating ghrelin, and feeding determines plasma ghrelin-like immunoreactivity levels in humans. J Clin Endocrinol Metab. 2001;86:4753-8.

30. Kelishadi R, Hashemipour M, Mohammadifard N, et al. Shortand long-term relationships of serum ghrelin with changes in body composition and the metabolic syndrome in prepubescent obese children following two different weight loss programmes. Clin Endocrinol (Oxf). 2008;69:721-9.

31. Garcia JM, Iyer D, Poston WSC, et al. Rise of plasma ghrelin with weight loss is not sustained during weigth maintenance. Obes Res. 2006;14:1716.

32. Cummings DE, Purnell JQ, Frayo RS, et al. A preprandial rise in plasma ghrelin levels suggests a role in meal initiation in humans. Diabetes. 2001;50:1714-9.

33. Karamanakos SN, Vagenas K, Kalfarentzos F, et al. Weight loss, appetite suppression, and changes in fasting and postprandial ghrelin and peptide-YY levels after Roux-en-Y gastric bypass and sleeve gastrectomy: a prospective, double blind study. Ann Surg. 2008;247:401-7.

34. Rodieux F, Giusti V, D'Alessio DA, et al. Effects of gastric bypass and gastric banding on glucose kinetics and gut hormone release. Obes Res. 2008;16:298-305.

35. Harada I, Nakahara T, Yasuhara D, et al. Obestatin, acyl ghrelin, and des-acyl ghrelin responses to an oral glucose tolerance test in the restricting type of anorexia nervosa. Biol Psychiatry. 2008;63:245-7.

36. Epelbaum J, Bedjaoui N, Dardennes R, et al. Role of the ghrelin/ obestatin balance in the regulation of neuroendocrine circuits controlling body composition and energy homeostasis. Mol Cell Endocrinol. 2010;314:244-7.

37. Akamizu T, Shinomiya T, Irako T, et al. Separate measurement of plasma levels of acylated and desacyl ghrelin in healthy subjects using a new direct ELISA assay. J Clin Endocrinol Metab. 2005;90:6-9. 